\title{
Adolescents' Communication Styles for Learning About Birth Control from Mass Media
}

\author{
Rocco De Pietro, Ph.D. \\ Noreen Clark, Ph.D.
}

\begin{abstract}
This article is an exploratory study to identify discrete factors which predict the way in which adolescents are likely to communicate about birth control after receiving information from a mass medium is described. First, we discuss styles of interacting with others regarding information from mass media which have been described in previous research. Five styles are identified: media-oriented, peer-oriented, home-oriented, professionally-oriented, and multi-source oriented. Next, we discuss categories of factors which theoretically should distinguish among the five communication styles. These factors are drawn from theories and previous research in communication and social learning. Using data collected from a random sample of 100 adolescents, we then test the predictive ability of categories of factors. Discriminant analysis is used to determine which factors within categories are most predictive of style and which best discriminate between styles. Eight factors are identified which predict $75 \%$ of all communication styles. The factors are sex, race, giving advice about sex, seeking information about birth control, dating rules regarding where one can go, dating rules regarding behavior on dates, feelings of pride, and feelings of popularity. Finally, we discuss the significance for practitioners of study findings and of predicting adolescents' communication styles.
\end{abstract}

Recent literature on acquisition of information about birth control from mass media suggests that adolescents have different communication styles that significantly affect their knowledge levels. Social interaction with significant others, such as parents and friends, is not only associated with high levels of birth control knowledge to begin with but also with knowledge gain from exposure to a magazine on birth control.' These findings are very much in keeping with the more general literature on the effects of mass media on children and adolescents which suggests that social

Rocco De Pietro and Noreen Clark are with the Department of Health Behavior and Health Education. The University of Michigan. Ann Arbor, MI. 
interaction during or following exposure to media leads to greater information gain. ${ }^{2 \cdot 5}$ For example, television viewing studies show that while few parents discuss program content with their children, ${ }^{6}$ those that do can influence their children's attention to and comprehension of certain content.' In addition, interaction with others while attending to media can facilitate the processing of novel stimuli. Social learning theory suggests that acquiring novel information, such as that related to birth control, requires more mental elaboration than routine information. ${ }^{8}$ Communication with others, such as family members and friends, aids this mental elaboration process."

If communication styles are highly associated with acquiring information about birth control from mass media, then it would be helpful to health planners and educators concerned with the delivery of birth control information to know something about the predictors of adolescents' styles. This knowledge could be used to develop strategies to reach different groups of adolescents as well as to arrive at a suitable approach to inform and educate them at family planning clinics and other settings. In this article, we examine some of the predictors of communication styles regarding information about birth control received from mass media. We also explore some ways in which these predictors might be used by health professionals as a diagnostic tool.

\section{THE CONCEPT OF COMMUNICATION STYLES}

Communication style is a concept that takes into account social interaction regarding information received from mass media. Communication styles have been conceptualized as being interactant or non-interactant, ' depending on whether they involve communication with others in the process of media use. Interactant forms should not be confused with incidental or haphazard acquisition of information from mass media such as the casual noting and recalling of messages from a medium, on a topic, during a recent time period. ${ }^{10}$ Interactant styles are purposeful and likely to occur when the information presented is complex, novel, salient to the individual, and likely to be positively reinforced by significant others including family or friends.

Adolescents who have non-interactant styles purposely acquire information from mass media without assistance from anyone. Non-interactant styles are likely to occur when content is very simple, requiring very little if any mental elaboration; when the content is perceived as somewhat boring or trivial (non-salient); when the content is highly personal or even taboo; or when 
negative reinforcement of learning behaviors by significant others is an expected outcome.

Interactant communication styles differ depending on the number and kinds of sources contacted. Previous research has enabled us to distinguish between individuals who communicate primarily with a single cluster of sources, such as the mass media, family members and relatives, or friends, and those who contact more than one cluster, e.g., family and friends. ' We refer to the former as single cluster styles and to the latter as multi-cluster styles. Some examples of single cluster styles are a home-oriented style, where communication is primarily with immediate family members and relatives; and a media-oriented style, where communication is exclusively with mass media. Another single cluster style is one that involves communication with technical experts about some topic. Such a style might be referred to as a professionally-oriented style. In the area of birth control communication, adolescents who have this style might communicate more with teachers, counselors, or the school nurse than with others.

A multi-culster style involves communication with more than one cluster without the majority of one's communication occurring with any particular cluster. This is analogous to the liaison role in network analysis where the person has communication linkages with more than one group without having the majority of these linkages with either group. "We refer to the holder of such a style as multi-source-oriented. In the process of considering information presented in mass media, the multisource-oriented adolescent contacts multiple clusters, such as family, friends, and professionals without establishing communication linkages primarily with any one group. Those with a multi-source style have more potential opportunities than others to acquire information because they have a variety of sources to help with processing and elaborating information.

An important non-interactant communication style is a mass media style. A person who has such a style uses mass media exclusively information without communicating with anyone about the content. The person might consult other mass media for further information but does not consult others, even for referral to these sources.

The concept of interactant and non-interactant communication styles is important because it describes the different ways in which people use mass media for acquiring information. It also illuminates the importance of the social context for knowledge acquisition, and how some learners from mass media use other people in processing and elaborating on information 
In their study of adolescents' communication styles and learning about birth control, De Pietro and Allen found evidence for these five theoretical styles.' Of their probablity sample of 100 adolescents, $37 \%$ were peer-oriented, $32 \%$ media-oriented, $19 \%$ home-oriented, $11 \%$ multi-source-oriented, and $1 \%$ professionallyoriented.

\section{THEORETICAL LINKAGES BETWEEN PREDICTORS AND COMMUNICATION STYLES}

Adolescents who interact with multiple clusters of individuals, including parents, friends, and professionals regarding content of mass media (the multi-source style), theoretically should be distinguished on a number of factors from adolescents who do not communicate with anyone (media-oriented style). Just examining these two "extreme" groups, for a moment, it is quite reasonable to expect, for example, that the multi-source-oriented have more positive sexual and birth control attitudes than media-oriented ones due to the greater potential they have to receive encouragement from many sources.

In stating some of the theoretical linkages between style and predictors, we recognized that our approach was exploratory. We were searching for factors which might distinguish between communication styles, that subsequently we could examine empirically. In our search, we were guided by theory and findings from previous research, primarily that of De Pietro and Allen.'

\section{Demographic Factors}

Sex, race, and presence of siblings in the home have been found to be significantly related to communication styles.' Females are more likely to be multi-source-oriented, whereas males are more likely to be media-oriented. With respect to race, non-whites are more likely to be found among the home-oriented and multisource-oriented styles, whereas whites are more likely to be found among the peer-oriented and media-oriented styles. Adolescents without siblings are more likely to have home-oriented styles than adolescents with them. Somewhat surprisingly, age was not found to be significantly related to communication style.

\section{Adolescents' Perceptions, Attitudes, and Behaviors}

Social learning theory suggests some ways in which adolescents' attitudes could be related to communication styles about birth 
control. The theory suggests that the anticipation of positive reinforcement from self and significant others can have a direct bearing on patterns of social interaction. ${ }^{8}$ For example, adolescents who are generally supportive of their own efforts to gain information about sexuality and birth control would be more likely than others to have interactant communication styles, especially one involving communication with multiple clusters of individuals (e.g., the multi-source style). In their efforts to find out more about birth control, these adolescents would have more sources of positive reinforcement, which in cyclical fashion would lead to more self-approval for information exchange. If the source of this perceived support is mainly from peers, a peer-oriented style would be predicted. If it is from multiple sources, a multisource-oriented style would be the prediction. The perceptions of significant others, such as one's peer group members, have been found to be a powerful determinant of adolescents' behavior in the sexual domain. ${ }^{213}$.

Some authors report a positive relationship between communication and sexual behavior. For example, some have found that sexually active, adolescent females tend to have poor communication relationships with parents. ${ }^{1+}$ This finding suggests that such females are less likely than others to have a homeoriented style. Our data do not support this finding.

\section{Feelings of Self-esteem}

Self-esteem is thinking well of oneself. ${ }^{15}$ Adolescents with high self-esteem are ones who appreciate their own merits, have selfrespect, and consider themselves persons of worth. During adolescence, relationships with parents have an important impact on self-esteem. For example, adolescents who can discuss important matters with their parents have available to them a powerful form of acceptance for their ideas. Such acceptance and recognition by adults can contribute greatly to feelings of selfworth. "Parents can also be helpful in guiding their adolescents through periods of rapid and intense personal change that of ten accompany the onset of puberty. One would theorize that adolescents who are high in self-esteem are more likely than others to be home-oriented in their communication style. This style suggests that they can communicate with parents in areas that are important to their growth and development, i.e., sexuality and birth control. Of course, other aspects of the home environment will affect communication as well. 


\section{Rules Coverning Dating Behavior}

Adolescents receive from parents very little socialization about sex that is conducive to effective role playing. ${ }^{17}$ Perhaps for this reason, many parents rely on rules to guide their adolescents' dating behavior during the early years. Dating rules might be viewed as a mechanism that parents use to protect their adolescents from potentially risky situations outside their immediate control. Rules governing dating behavior are likely to vary depending on the influence that parents feel others have on their children's sexual behavior. Parents who view their children as home centered are likely to feel that their adolescents do not need very many dating rules. Adolecents with home-oriented communication styles about birth control might be found in this group. However, parents who perceive that their adolescents are heavily involved with peer groups might feel the need to protect them with various rules. Adolescents with peer-oriented and multisource styles might be found among this group.

\section{Social Competency}

Competency is an important personal attribute that affects the extent to which a person will be liked ${ }^{18}$ While the general notion of competency pertains to the capacity to perform well on a variety of tasks over time, social competency pertains to performance on tasks that involve interaction with others. For adolescents, this might mean being a good athlete, dancer, listener, or companion. Adolscents who are socially competent should be liked by others because they can do things that are highly valued within the peer environment. We expected that these adolescents would be highly represented among those persons with peer-oriented or multi-source-oriented styles.

In selecting the five categories of predictors discussed above, our ultimate objective was to arrive at a discrete set of factors which predict communication styles. Data describing communication styles of adolescents, available from the previously cited De Pietro and Allen study, ' enabled us to examine empirically the theoretical relationship between the styles and some of their predictors.

\section{RESEARCH METHODOLOGY}

The study sample consisted of 100 randomly selected adolescents between the ages of 13 and 17 from a middle-sized 
industrial city in the Midwest. Of the sample, $61 \%$ were females, $58 \%$ between the ages of 13 and 15 , and $76 \%$ non-white. These 100 individuals were "target teens" in a field study that was designed to test a model of delivering information on birth control to adolescents. The field experiment sample consisted of about 200 adolescents selected randomly from households in and around the city and randomly assigned to experimental or control groups. The current analysis is based only on the "target teens" that formed the experimental group. The fact that a local research organization maintains a complete, up-to-date listing of households in the community, enabled generation of a truly random sample for the field experiment and, therefore, the present study. The response rate for the study was $70 \%$. Of the $30 \%$ refusals, a slight majority are males.

As part of the field experiment, "target"adolescents were asked to read a magazine on human sexuality and birth control, called Making Choices, which was prepared especially for them Adolescents were asked simply to read the magazine (they had eight weeks to read it before the second interview) and share information with others if they so desired. They were not instructed to communicate with others or rewarded for doing so in any way. The objective of the field experiment was to capture differences in social process involved in learning from the magazine.

\section{Measure of Communication Styles}

The measure of communication style used in the field experiment and the present study is described in De Pietro and Allen (1982). It consisted of self-reports of communication behavior during the process of learning about birth control from the magazine. Those who read the magazine but did not discuss its content with anyone were classified as possessing a media-oriented style. Those who chose not to discuss the contents with others were asked to place a check next to the persons they spoke with. Response choices included mother, father, brothers, sisters, relatives, best friend of the same sex, best friend of the opposite sex, school friends, non-school friends, other teens, teachers, counselors, doctors, and other adults

Adolescents who had the majority of communication linkages (over half) with family members and relatives were classified as having a home-oriented style. Those who had the majority of linkages with friends and own-age peers were classified as having a peer-oriented style. Those who had the majority of their linkages with professionals or experts were classified as having a 
professionally-oriented style. Finally, those who had multiple linkages with others including family members and relatives, friends and own-age peers, and professionals or experts, but the majority of them with no single cluster, were classifed as having a multi-source-oriented style.

\section{MEASURES OF PREDICTIVE FACTORS}

For the present study, questionnaire data from the field experiment were used to develop several measures of predictors of style the self esteem measureconsists of a four-point scale on which respondents are asked to indicate the extent to which they agree or disagree with 10 belief statements. To measure respondents' perceptions, attitudes, and behaviors related to sexuality and birth control, a series of items was used which required that each adolescent "place" him or herself somewhere along a 10-point scale. Items referred to knowledge, practices, and views regarding birth control and sexuality, giving advice to other adolescents, and seeking information on the topics. Another series of items asked adolescents first to identify the characteristics of social competence and then rate themselves on each item. These items related to the extent of the popularity, grades, dancing, sports, being sought for advice, attractiveness to the opposite sex, dependability, being a trend setter, influencing others, and being shown respect. To measure respondents' self-èsteem, we used Rosenberg's index of 10 belief items. ${ }^{15}$ Finally, as measures of dating rules items were used which related to limits set by parents. These items referred to curfews, amount of dating, dating activities, being at home alone with a date, where one can go, and who one can date.

\section{ANALYSIS OF RESULTS}

We approached the analysis by first entering data from questionnaire items representing each group of predictors into a multivariate analysis to determine which were significantly related to communication styles, i.e., showed the best discrimination. We then used this information to plan a final analysis in which the best predictors among items from the five groups were entered into a multivariate analysis. The method permits any number of discriminating variables, provided that it is less than the total number of cases minus two and that there are at least two cases per group. ${ }^{19}$ While our data meet the assumptions of the model. 
our relatively small sample size is reason to be careful in the interpretation of results. Some kind of cross-validation of findings is necessary before we become firmly committed to results.

The discriminant analysis provides us with two useful kinds of information. First, the standardized discriminant coefficients tell us which predictor variables contribute the most to determining the scores on the function for each category of the variable, communication style. The larger the magnitude of the coefficient, regardless of sign, the greater the predictor's contribution. This information is gleaned by reading up and down the columns in Table 1 . Second, the relative magnitudes of the standardized coefficients can also be used to determine the variables that make the greatest contribution to the discrimination between and among groups. ${ }^{20}$ The larger the difference between the values of two coefficients, the better they discriminate between two groups of the dependent variable. This information is found by reading across the rows of Table 1

The results of the multiple discriminant analysis show that eight factors are significantly related to communication styles when all are considered simultaneously. They are: sex, race, giving advice about sex, seeking information about birth control, dating rules regarding where one can go on dates, dating rules regarding how to behave on dates, pride, and feelings of success as indicators of self-esteem. While these eight factors were found to be significant in a multivariate analysis, the majority of them also have signifi$c$ ant independent relationships with communication styles. (For sex of respondents, $x^{2}=8.75, p<.03 ;$ race, $x^{2}=14.08 ; p<.00$; give advice about sex, $x^{2}=5.97, p<11$; seek information about birth control, $x^{2}=7.90, p<.05$; dating rules - where can go, $x^{2}=13.36, p<.00$; dating rules-behavior on dates, $x^{2}=1.90, p<60$; selfesteem-pride, $x^{2}=17.59, p<.01$; social competency-popularity; $x^{25} 16.60, p<.06$. Interestingly, we found no significant relationship between adolescents' sexual behavior and their communication styles about birth control $\left(x^{2}=1.48, p<.69\right)$.

\section{Best Contributors to Each Communication Style}

This analysis reports which factor or predictor contributes the most to the score of each communication style.

For the home-oriented style, we see that self-esteem, giving advice about sex, and rules regarding behavior on dates make the greatest contribution to calculating the discriminant score for that group. The style is associated with high self-esteem, giving little advice about sex, and having rules about how to behave on dates.

For the peer-oriented style, race, self-esteem (pride), and social 


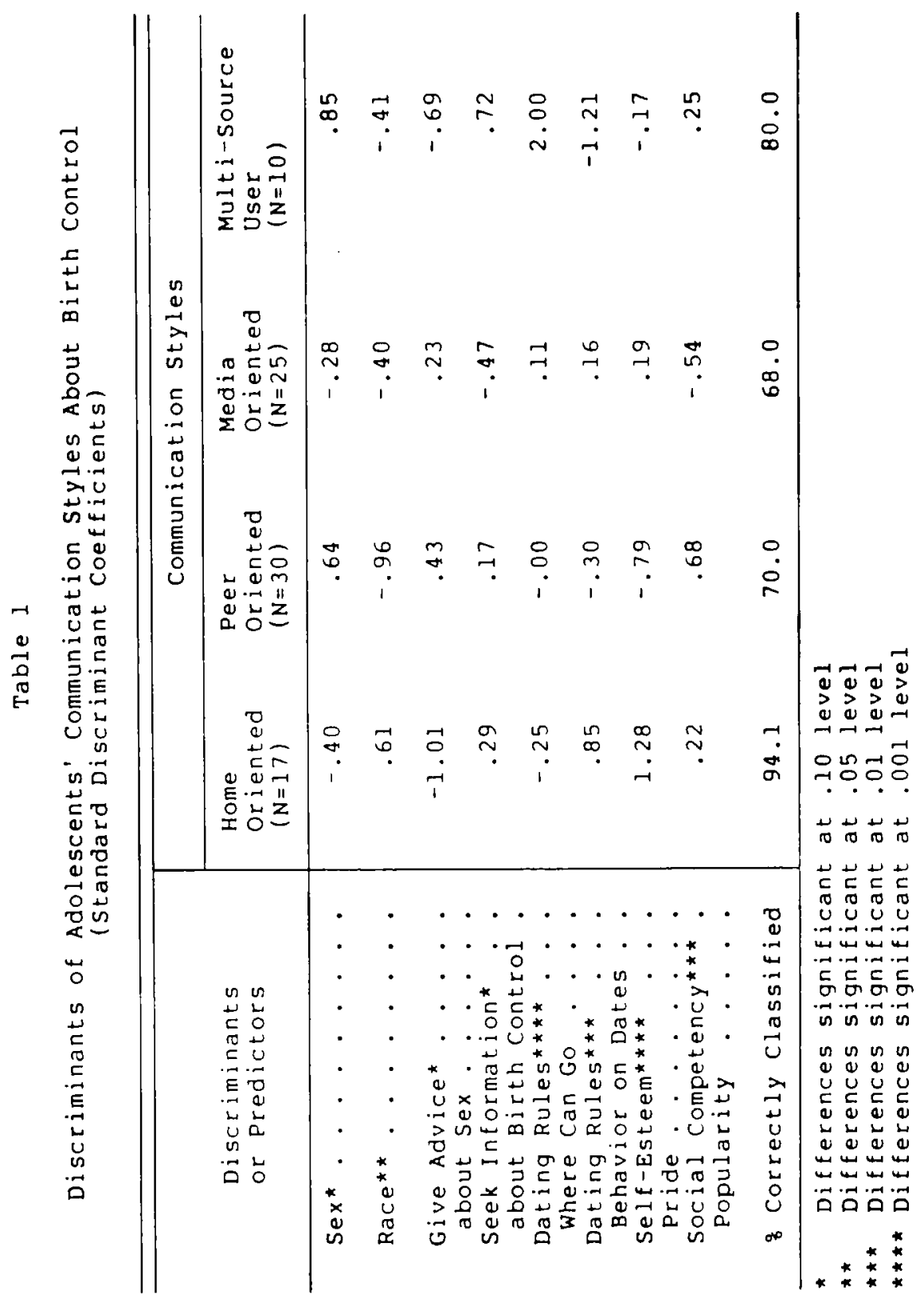


competency (popularity in school) make the greatest contribution. Adolescents with this style are more likely to be white, relatively low in self-esteem, and somewhat high in popularity at school.

For the media-oriented style, social competency (popularity in school), and seeking birth control information make the greatest contribution. Adolescents with this style are more likely to feel that they are unpopular in school and are very unlikely to seek birth control information. None of the predictors contribute very much to this group.

For the multi-source-oriented style, dating rules make the greatest contribution, including where to go on dates and behavior on dates. Interestingly, the multi-source-oriented adolescent is much more likely to have rules regarding where to go on dates as opposed to rules regarding actual dating behavior. Perhaps the parents of these adolescents believe that it is much easier to control the former than the latter. Sex, seeking information about birth control, and giving advice about sex also make important contributions to the score of this group. The multi-source-oriented adolescent is much more likely to be female and seek information about birth control. However, she is not very likely to give advice about sex.

\section{Factors Which Best Discriminate between Styles}

Let's look again at Table 1 to determine which communication styles are best discriminated by each predictor. Here we look for large differences between the standardized coefficients for each factor or predictor.

Race best discriminates between peer-oriented and homeoriented adolescents with the latter composed of primarily nonwhites.

Giving advice about sex best discriminates between adolescents with adolescents with peer-oriented styles and those with homeoriented ones, the former much more likely to be the advice givers on this topic.

Seeking birth control information best discriminates between the multi-source style and media-oriented styles with the former being the information seekers.

Dating rules discriminate between communication styles very differently. Rules regarding where adolescents can go on dates best discriminates between the multi-source-oriented, who have such rules, and adolescents with home-oriented styles who do not. Rules regarding behavior on dates best discriminates between the same styles, with the adolescents with home-oriented styles having the rules in this instance. 
Of the self-esteem factors, pride best discriminates between adolescents with peer-oriented styles and those with homeoriented ones, the latter experiencing a much greater sense of it.

Of the social competency factors, popularity in school best discriminates between peer-oriented and media-oriented styles, with adolescents who hold the latter not feeling very popular with their peers. This finding suggests that adolescents with media-oriented, or non-interactant styles might be the "loners" at school and in other social settings. Some simply lack the opportunity to interact with peers in the process of acquiring new material.

The eight factors were able to predict about $75 \%$ of all communication styles. The precent correctly classified for each style are: home-oriented, $94.1 \%$; peer-oriented, $70.0 \%$; mediaoriented, $69.0 \%$; and multi-source-oriented, $80.0 \%$

\section{DISCUSSION AND CONCLUSIONS}

These findings lend support to the idea that it is possible to predict an adolescent's communication style after receiving information about birth control from mass media, on the basis of a few items of data that are relatively easy to observe and collect. Further, the study suggests that some predictors are especially effective in discriminating between given styles. The implications of such findings for practice are significant.

Family planning and health education and service to adolescants have become prevalent and sophisticated in recent years. Nonetheless, few practitioners and programs are as effective as we might hope in communicating with young people. Part of the problem is knowing how to frame one's appeal to adolescents. If if were possible to predict the way in which a person is most likely to acquire information and communicate about new concepts, practitioners could select appropriate educational or counselling strategies. These would enhance information gains from mass media, in accordance with individual communication style. One would envision, for example, that prior to a consultation with a physician, nurse, family planner, health educator, or other practitioner, an adolescent would complete a short questionnaire. The questionnaire would be comprised of items similar to those factors used in this study. Using a simple scoring system, the practitioner would make a judgment regarding the adolescent's likely commuication style. Based on the style, the practitioner would frame information, place emphasis, and suggest additional activities to support learning from written material.

At the first level, the score would distinguish interactant from 
non-interactant learners. At the second level, specific communication styles would be identified. For those with a media-oriented, or non-interactant, style, for example, activities could be offered which afford the young person the opportunity to interact with others to communicate about new family planning concepts. Such interaction appears necessary for mental elaboration required to understand complex ideas. A mass media-oriented client might be offered the chance to view a videotape or film expanding on information and ideas presented in printed material. He may be offered more extensive individualized counseling and the opportunity to discuss with the practitioner concepts presented in the media. He may receive frequent reassurance in his effort to acquire information and be encouraged by the practitioner to call for further consultation and with any questions he may have.

For clients with single cluster interactant styles, activities could be offered which reinforce the way in which they are predisposed to communicate. For example, the practitioner might present a young person likely to be peer-oriented with printed material about family planning and encourage her to take part in organized group sessions with young women. Here she could be offered the chance to discuss material further and share her views with others her own age. On the other hand, a client likely to be home-oriented could be encouraged to discuss concepts further with his family members and to telephone the clinic with any questions he or they might have. Materials may even pose questions or points for discussion with parents or siblings

Clients with multi-cluster interactant styles, the multi-sourceoriented, could be offered a range of options. These adolescents, who already use various sources of information, could be reinforced in their efforts to communicate with family and peers and use media

Through testing, it is very likely that this preliminary work can be refined and made more precise. The intention of developing a reliable set of predictors of style would in no way be an attempt to stereotype adolescents receiving family planning information. Rather, identification of communication styles would be an effort to enhance communication between practitioner and client, to make optimum the chance that an adolescent receives and internalizes family planning information. It may well be that a precise set of predictors would also lead to general improvement in verbal messages and printed material targeted towards groups of adolescents. One can infer from this exploratory study that the time may not be too distant when educators can discriminate communocation styles and tailor-make family planning information. 


\section{REFERENCES}

1 De Pietro RA. Allen RI Communcation styles and learning about birth control Adolescence (In press)

2. Corder-Bols. CR. Mediation the role of signific ant others lournal of Communication $30(3)-106-118,1980$.

3 Robertson TS: Parental mediation of television effects. Journal of Communication $29(1): 12-25,1979$

4. Saloman G: Interaction of Media. Cognition and Learning. San Francisco Iossey-Bass 1979

5. Clarke P. De Pietro RA. Delivering information on human sexuality and birth control to teenagers. the lest of a social comparison approach A report to the C.S. Mott Foundation, Department of Communication. The University of Michigan, Ann Arbor Michigan, 1980

6. Greenberg BS, tricson PM. Vlahou $M$ Children's television behaviors as perceived by mother and child Michigan State University. 1971 (LRIC Document Reproduction Service No. FD 058750 )

7 Cross L. Morgan $M$ Television and enculturation In I Dominick and IK Fletcher (Eds), Broadcasting Research Methods: A Reader. Boston: Allyn and Bacon, in press

8 Bandura A: Social Learning Theory. Morrstown. NJ: Ceneral Learnong Press. 1971

9 Greeno IC. Language understanding and learning A paperpresented at the Symposium on Individual Differences, Cognition, and Learning. American Association for the Advancement of Science, Denver, Colorado, 1977

10. Clarke P. Kline FC. Media effects reconsidered Communication Research 1:224-240. 1974.

11 Farace RV, Monge PR, Russell HM: Communicating and Organizing. Reading, MA Addian-Weslev, 1977

12 Mirande $M$. Reference group theory and adolescent sexual behavior lournal of Marriage and the Family 30:572-577, 1968

13. Schulz B. Bohrnstedt GW. Borgatta EF. Evans RR: Explainıng permarital sexual intercourse among college students: a causal model. Social Forces 56.148-165, 1977

14 Chilman: CS. Adolescent Sexuality in a Changing American Society. DHFW Publication. No $(\mathrm{NIH}) 79-1226$

15 Rosenberg $M$ : Conceiving the Self. New York Basic Books, Inc 1979

16. Douvan E, Adelson 1. The Adolescent Experience. New York: Wiley, 1966

17. Nye I. Berardo FM: The Family lis Structure and Interaction. New York Macmullan Publishing Co., 1973

18. Aronson $\mathrm{F}$ : The Social Animal. San Francisco WH Freeman and Co, 1972

19. Klecka R: Discriminant Analysis. Beverlev Hills. Sage Publications, 1980

20. Pedhazur EJ. Multiple Regression in Behavioral Research. New York. Holt. Runehart and Winston, 1982. 